\title{
Numerical Study on Gas Flow through a Micro-Nano Porous Media
}

S. ABBASBANDY*

Department of Mathematics, Imam Khomeini International University, Ghazvin, 34149-16818, Iran

(Received May 10, 2011; revised version September 17, 2011; in final form October 11, 2011)

\begin{abstract}
The present research examines the unsteady isothermal flow of a gas through a semi-infinite micro-nano porous medium, a nonlinear boundary value problem on semi-infinite interval. This problem is solved by two different methods and compare their results with solution of other methods is compared. Also through the convergence of these methods, the accurate initial slope $y(x)$ with good capturing the essential behavior of $y(x)$ is obtained.
\end{abstract}

PACS: 02.70.Bf, 68.47.-b, 87.10.Ed, 95.90.+v

\section{Introduction}

There are many problems in science and engineering arising in unbounded domains which can be modeled by singular and nonsingular boundary value problems. The application of these problems involves chemical kinetics, astrophysics, experimental and mathematical physics, nuclear charge in heavy atoms, thermal behavior of a spherical cloud of gas, thermodynamics, population models, fluid mechanics and many other topics. Several techniques including decomposition, variational iteration, finite difference, polynomial spline, homotopy analysis method, and shooting methods have been developed for solving such problems.

Gas-solid processes like adsorption, are used in the chemical industries to separate solutes from a fluid stream. Transport phenomena and diffusion in micro-nano porous materials have attracted the researchers' attention for a long time. The modeling of gas flow through a porous media is quite valuable because of its importance in investigating gas-solid processes. The flow of gas through a semi-infinite porous medium initially fills with gas at a uniform pressure $P_{0}>0$, at time $t=0$, the pressure on the outflow face is suddenly reduced from $P_{0}$ to $P_{1} \geq 0\left(P_{1}=0\right.$ in the case of diffusion into a vacuum $)$ and thereafter, maintained at this lower pressure. The unsteady flow of gas in a porous medium is modeled by a nonlinear partial differential equation [1-3] as follows:

$$
\nabla^{2}\left(P^{2}\right)=(2 \Phi \mu / k) \frac{\partial P}{\partial t}
$$

where $P$ is the pressure within porous medium, $\Phi$ is the porosity, $\mu$ is the viscosity, $k$ is the permeability and $t$ is the time.

In the one-dimensional medium extending from $z=0$ to $z=\infty$, Eq. (1) reduces to

\footnotetext{
* e-mail: abbasbandy@yahoo.com
}

$$
\frac{\partial}{\partial z}\left(P \frac{\partial P}{\partial z}\right)=(\Phi \mu / k) \frac{\partial P}{\partial t},
$$

with the boundary conditions

$$
\begin{aligned}
& P(z, 0)=P_{0}, 0<z<\infty ; \\
& P(0, t)=P_{1}\left(<P_{0}\right), 0 \leq t<\infty .
\end{aligned}
$$

Now, by using the new independent variable [4]:

$$
x=\frac{z}{\sqrt{t}}\left(\frac{A}{4 P_{0}}\right)^{1 / 2},
$$

and the dimension-free variable $y$, defined by

$$
y(x)=\alpha^{-1}\left(1-\frac{P^{2}(z)}{P_{0}^{2}}\right),
$$

where $A=\Phi \mu / k$ and $\alpha=1-\frac{P_{1}^{2}}{P_{0}^{2}}$, a similarity solution is obtained. In terms of these variables, the problem takes the form (unsteady gas equation)

$$
\begin{gathered}
y^{\prime \prime}(x)+\frac{2 x}{\sqrt{1-\alpha y(x)}} y^{\prime}(x)=0, \\
x>0, \quad 0<\alpha<1 .
\end{gathered}
$$

The typical boundary conditions imposed by the physical properties are

$$
y(0)=1, \quad \lim _{x \rightarrow \infty} y(x)=0 .
$$

A substantial amount of numerical and analytical work has been invested so far on this model [1-3]. The major drawback of these methods is huge computational work. This problem (5) was handled by Kidder [2]. Also a perturbation technique is carried out to include terms of second order, and the convergence of the obtained expansion is guaranteed through physical properties of $y(x)$, and it is shown that the complexity of the calculations increases rapidly with increasing order of the terms. Wazwaz [5] proposed modified Adomian decomposition method, and $[M / M]$ Padé approximation method for this equation, in spite of the fact that $M$ is the degree of polynomial in series solution.

Aslam Noor and Mohyud-Din [6] considered modified variational iteration method for this equation. Parand 
et al. [7] solved this problem by using pseudospectral method with rational Chebyshev and modified generalized Laguerre functions. Taghavi et al. [8] solved it by Lagrangian method based on modified generalized Laguerre functions. Khan et al. [9] used modified Laplace decomposition method (MLDM) coupled with Padé approximation technique to solve this equation. MohyudDin et al. [10] applied the coupling of He's polynomials to the correct functional of variational iteration method to solve the problem (5). This equation has been recently considered by Rezaei et al. [11] with the orthogonal rational Legendre and Sinc functions with using pseudospectral method.

In this paper most of the obtained results of $y^{\prime}(0)$ are the same, because the researcher used Padé approximation methods with different functions. See Table I to compare obtained results. In this table, $N$ is the degree of orthogonal polynomial used in the method and MGLFM, RCM, MGL, SINC and RLP are the abbreviation for modified generalized Laguerre function method, rational Chebyshev method, modified generalized Laguerre function method, pseudospectral method based on sinc function, and rational Legendre pseudospectral method, respectively. More results can be found for other values of $\alpha$ in the mentioned papers.

Values of $y^{\prime}(0)$ for $\alpha=0.5$.

TABLE I

\begin{tabular}{|c|c|c|}
\hline Paper & Method & $y^{\prime}(0)$ \\
\hline \multirow[t]{2}{*}{ Wazwaz [5] } & Padé $_{[2 / 2]}$ & -1.373178096 \\
\hline & Padé $_{[3 / 3]}$ & -1.025529704 \\
\hline \multirow[t]{2}{*}{ Aslam Noor [6] } & Padé $_{[2 / 2]}$ & -1.373178096 \\
\hline & Padé $_{[3 / 3]}$ & -1.025529704 \\
\hline \multirow[t]{4}{*}{ Parand [7] } & $\operatorname{MGLFM}(N=6)$ & -1.36417503 \\
\hline & MGLFM $(N=7)$ & -1.38213483 \\
\hline & $\operatorname{RCM}(N=6)$ & -1.10805718 \\
\hline & $\operatorname{RCM}(N=7)$ & -1.26259357 \\
\hline \multirow[t]{2}{*}{ Taghavi [8] } & MGL $(N=6)$ & -1.37310852 \\
\hline & MGL $(N=7)$ & -1.37317352 \\
\hline \multirow[t]{2}{*}{ Khan [9] } & Padé $_{[2 / 2]}$ & -1.373178096 \\
\hline & Padé $_{[3 / 3]}$ & -1.025529704 \\
\hline \multirow[t]{2}{*}{ Mohyud-Din [10] } & Padé $_{[2 / 2]}$ & -1.373178096 \\
\hline & Padé $_{[3 / 3]}$ & -1.025529704 \\
\hline \multirow[t]{8}{*}{ Rezaei [11] } & $\operatorname{SINC}(N=6)$ & -1.188228002 \\
\hline & $\operatorname{SINC}(N=8)$ & -1.188625937 \\
\hline & $\operatorname{SINC}(N=16)$ & -1.188689251 \\
\hline & $\operatorname{SINC}(N=32)$ & -1.188692320 \\
\hline & $\operatorname{RLP}(N=6)$ & -1.188610520 \\
\hline & $\operatorname{RLP}(N=8)$ & -1.188650543 \\
\hline & $\operatorname{RLP}(N=16)$ & -1.188677428 \\
\hline & $\operatorname{RLP}(N=32)$ & -1.188687197 \\
\hline
\end{tabular}

It will be shown that the obtained results by Rezaei et al. [11] are more accurate than others. The aim of this paper is to obtain more accurate results by using two different approaches which are implicit finite-difference scheme known as the Keller-box method [12, 13] and the shooting method [13].

\section{Solution procedures}

\subsection{Finite-difference method}

To solve the differential Eq. (5) which is subject to boundary conditions (6), the first Eq. (5) is converted into a system of two first-order equations, and the difference equations are then expressed by using central differences. For this purpose, the new dependent variable $w(x)$ is introduced, so that Eq. (5) can be written as

$$
\begin{aligned}
& y^{\prime}(x)=w(x), \\
& w^{\prime}(x)=\frac{-2 x w(x)}{\sqrt{1-\alpha y(x)}} .
\end{aligned}
$$

Now, by using the Taylor series expansion of $\frac{1}{\sqrt{(.)}}$, Eq. (8) is changed to

$$
\begin{aligned}
& w^{\prime}(x)=-2 x w(x)\left[1+c_{1} \alpha y(x)+c_{2} \alpha^{2} y(x)^{2}+\right. \\
& \left.\ldots+c_{m} \alpha^{m} y(x)^{m}\right],
\end{aligned}
$$

where $m$ is an arbitrary constant natural number and $c_{1}=\frac{1}{2}, c_{2}=\frac{3}{8}, c_{3}=\frac{5}{16}$, and so on. Now, we consider the segment $x_{j-1} x_{j}$, which is defined as:

$$
x_{0}=0, \quad x_{j}=x_{j-1}+h_{j}, \quad x_{J}=x_{\infty},
$$

where $h_{j}$ is $\Delta x$-spacing and $j=0,1, \ldots, J$ is a sequence number that indicates the coordinate location. In the Keller-box method, the finite-difference approximations to the ordinary differential Eqs. (7) and (9) are written for the midpoint $x_{j-1 / 2}$ of the segment $x_{j-1} x_{j}$ for $j=1,2, \ldots, J$ as

$$
\begin{aligned}
& y_{j}-y_{j-1}-\frac{1}{2} h_{j}\left(w_{j}+w_{j-1}\right)=0, \\
& w_{j}-w_{j-1}+h_{j} x_{j-1 / 2}\left(w_{j}+w_{j-1}\right) \\
& \quad \times\left[1+\frac{c_{1} \alpha}{2}\left(y_{j}+y_{j-1}\right)+\frac{c_{2} \alpha^{2}}{4}\left(y_{j}+y_{j-1}\right)^{2}\right. \\
& \left.\ldots+\frac{c_{m} \alpha^{m}}{2^{m}}\left(y_{j}+y_{j-1}\right)^{m}\right]=0 .
\end{aligned}
$$

The transformed boundary layer thickness $x_{J}$ is sufficiently large, so that it is beyond the edge of the boundary layer. The boundary conditions are

$$
y_{0}=1, \quad y_{J}=0 \text {. }
$$

Now by Newton's method, the nonlinear system is linearized (11), (12) and then, the obtained linearized difference equations can be solved by the block-elimination method as outlined by [3, 13], since the obtained system has block-tridiagonal structure. The obtained results for linear initial profile and stop criterion $10^{-8}$ on $y^{\prime}(0)$ with $h_{j}=h$ and $x_{\infty}=h J$ are shown in Table II. Table III shows the values of $y(x)$ by using perturbation technique [2], and Padé ${ }_{[3 / 3]}$ [5] for $\alpha=0.5$. Table IV shows that the initial slope $y^{\prime}(0)$ increases with the increase of $\alpha$. 
TABLE II

Values of $y^{\prime}(0)$ for $\alpha=0.5$ for different $x_{\infty}$.

\begin{tabular}{c|c|c|c}
\hline \hline$M$ & $J$ & $h$ & $y^{\prime}(0)$ \\
\hline 6 & 30 & $1 / 10$ & -1.1938713177347504 \\
& 60 & $1 / 20$ & -1.1922883859473303 \\
& 90 & $1 / 30$ & -1.1919951287546457 \\
& 120 & $1 / 40$ & -1.1918924798983241 \\
& 150 & $1 / 50$ & -1.1918449665961557 \\
& 300 & $1 / 100$ & -1.1917816140076933 \\
& 600 & $1 / 200$ & -1.1917657755896378 \\
& 1200 & $1 / 400$ & -1.1917618159682022 \\
8 & 1200 & $1 / 400$ & -1.1918059634783928 \\
10 & & $1 / 400$ & -1.1918126190284244 \\
12 & & $1 / 400$ & -1.1918137142588647 \\
14 & & $1 / 400$ & -1.1918139058828805 \\
16 & & $1 / 400$ & -1.1918139409674497 \\
18 & & $1 / 400$ & -1.1918139476201857 \\
20 & & $1 / 400$ & -1.191813948917348 \\
20 & 2000 & $1 / 40$ & -1.1919230334799826 \\
& 5000 & $1 / 100$ & -1.1918118315451425 \\
& 10000 & $1 / 200$ & -1.1917959449569746 \\
30 & 50000 & $1 / 1000$ & -1.1917908612172277 \\
30 & 5000 & $1 / 40$ & -1.1919230338118607 \\
30 & 10000 & $1 / 100$ & -1.1918118318711821 \\
30 & 50000 & $1 / 1000$ & -1.1917908615421229
\end{tabular}

TABLE IV

Initial slopes $y^{\prime}(0)$ for various values of $\alpha$, for $M=30$, $J=50000$.

\begin{tabular}{c|c|c|c}
\hline \hline$\alpha$ & Padé $_{[2 / 2]}$ & Padé $_{[3 / 3]}$ & Present method \\
\hline 0.1 & -3.556558821 & -1.957208953 & -1.139007356865483 \\
0.2 & -2.441894334 & -1.786475516 & -1.1504756481362894 \\
0.3 & -1.928338405 & -1.478270843 & -1.1629416335500269 \\
0.4 & -1.606856838 & -1.231801809 & -1.1766158581496988 \\
0.5 & -1.373178096 & -1.025529704 & -1.1917908615421229 \\
0.6 & -1.185519607 & -0.8400346085 & -1.2088944130728032 \\
0.7 & -1.021411309 & -0.6612047893 & -1.228598743345502 \\
0.8 & -0.8633400217 & -0.4776697286 & -1.2520835659442726 \\
0.9 & -0.6844600642 & -0.2772628386 & -1.281847782063427
\end{tabular}

\subsection{Shooting method}

The non-linear ordinary differential Eq. (5) together with the boundary conditions (6) are solved numerically using Nachtsheim-Swigert shooting iteration technique [14] (guessing the missing value) along with explicit Runge-Kutta initial value solver with stiffness detection capability by Mathematica Software.

As we know, in a shooting method, the missing initial condition at the initial point of the interval is assumed by user, and the differential equation is solved numerically as an initial value problem to the terminal point.
The accuracy of the assumed missing initial condition is then checked by comparing the calculated value of the dependent variable at the terminal point with its given value there. If a significant difference exists, then another value of the missing initial condition must be assumed and the process is repeated. This process is continued until the reasonable agreement between the calculated and the given condition at the terminal point is obtained within the specified degree of accuracy. For this type of iterative process, one naturally needs to a systematic way of finding each succeeding (assumed) value of the missing initial condition.

In this method the asymptotic boundary condition (6) is replaced by the condition that $y(x)=0$ to a sufficient degree of accuracy at $x=x_{\infty}$, where $x_{\infty}$ is the value of the independent variable at the edge of the boundary layer. The boundary-value problem is equivalent to the problem of finding a value of $y^{\prime}(0)$ for which the boundary condition at the edge of the boundary layer is satisfied. With the notation $z=y^{\prime}(0)$. It is observed that a small change $\Delta z$ in $z$ changes value of $y$ by the amount

$$
\frac{\partial y}{\partial z} \Delta z
$$

so, the necessary correction to the first approximation comes from the solution of the following linear equation:

$$
y(x)+\frac{\partial y}{\partial z} \Delta z=0,
$$

at $x=x_{\infty}$. The solution of (14) for $\Delta z$ can be obtained if we can evaluate $\frac{\partial y}{\partial z}$ at $x_{\infty}$. So, we must use differentiation of (5). With notation

$$
y_{z}=\frac{\partial y}{\partial z}, \quad y_{z}^{\prime}=\frac{\partial y^{\prime}}{\partial z}, \ldots
$$

and taking differentiation of (5) with respect to $z$, we have

$$
\begin{gathered}
y_{z}^{\prime \prime}(x)+\frac{2 x}{\sqrt{1-\alpha y(x)}} y_{z}^{\prime}(x)+y^{\prime}(x) y_{z}(x) \\
\times \frac{\alpha x}{\sqrt{[1-\alpha y(x)]^{3}}}=0,
\end{gathered}
$$

with initial conditions

$$
y_{z}(0)=0, \quad y_{z}^{\prime}(0)=1 \text {. }
$$

Given an arbitrary initial estimation of $z=y^{\prime}(0)$, subsequent values of $z$ can be computed by integrating Eqs. (5) and (15) and applying the Newton-Raphson method to obtain the corrections. For this reason, we need to the value of $x_{\infty}$ for getting the integration. After this, we show the tentative values of $x_{\infty}$ by $x_{\text {stop. }}$. We can start our process by a small value for $x_{\text {stop, }}$ and in each it-

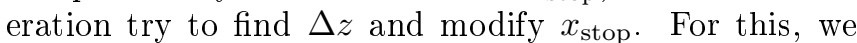
use from the boundary condition (6) at infinity and its derivative. That is, $\Delta z$ should be chosen so that both equations

$$
y(x)+y_{z}(x) \Delta z=0, \quad y^{\prime}(x)+y_{z}^{\prime}(x) \Delta z=0,
$$


TABLE III

Approximations of $y(x)$ for present methods in comparison with other method, for $\alpha=0.5, M=30, J=50000$.

\begin{tabular}{c|c|c|c|c}
\hline \hline$x$ & Kidder [2] & Padé $_{[3 / 3]}[5]$ & Rezaei [11] & Present method \\
\hline 0.1 & 0.8816588283 & 0.8979167028 & 0.8816297930 & 0.8813646572742952 \\
0.2 & 0.7663076781 & 0.7985228199 & 0.7666243657 & 0.7658288222744776 \\
0.3 & 0.6565379995 & 0.7041129703 & 0.6565307085 & 0.656000694626484 \\
0.4 & 0.5544024032 & 0.6165037901 & 0.5545172476 & 0.5538989492480791 \\
0.5 & 0.4613650295 & 0.5370533796 & 0.4628652574 & 0.4609427659504205 \\
0.6 & 0.3783109315 & 0.4665625669 & 0.3814536078 & 0.37798158206878896 \\
0.7 & 0.3055976546 & 0.4062426033 & 0.3095060359 & 0.3053523296040834 \\
0.8 & 0.2431325473 & 0.3560801699 & 0.2466311657 & 0.24295437638061612 \\
0.9 & 0.1904623681 & 0.3179966614 & 0.1927749120 & 0.19033421260699213 \\
1.0 & 0.1587689826 & 0.2900255005 & 0.1478076673 & 0.14677328712194618 \\
& & &
\end{tabular}

are satisfied at $x=x_{\text {stop }}$. Now, we have two equations with one unknown $\Delta z$, and by least squares method we can find it. Let $\delta_{1}=y\left(x_{\text {stop }}\right)+y_{z}\left(x_{\text {stop }}\right) \Delta z, \delta_{2}=$ $y^{\prime}\left(x_{\text {stop }}\right)+y_{z}^{\prime}\left(x_{\text {stop }}\right) \Delta z$, and the error $E=\delta_{1}^{2}+\delta_{2}^{2}$. It can be found that $E$ takes its minimum when $\Delta z$ is obtained by doing least squares method on Eqs. (17). In Fig. 1, the error $E$ is shown plotted as a function of $z$ for various values of $x_{\text {stop }}$.

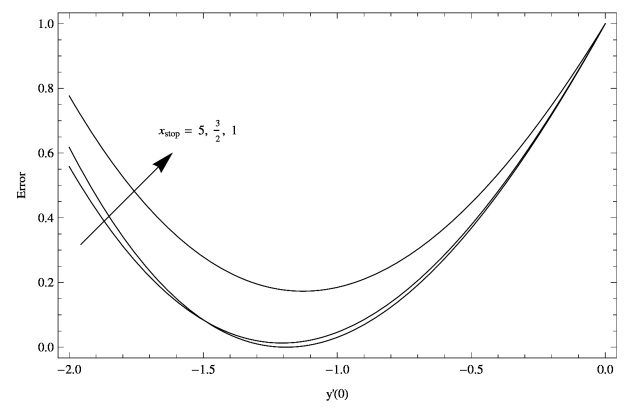

Fig. 1. Error as function of $y^{\prime}(0)$ for different values of $x_{\text {stop }}$.

Let $E_{\min }$ is the minimum of $E$ with respect to $z$. Now, in next step we want to decrease the value of $E_{\min }$. At first, we start with a small value for $x_{\text {stop }}$, say $x_{\text {stop }}=1$. A step size of $\Delta x=0.01$ was selected to be satisfactory for a convergence criterion of $10^{-8}$ in all cases (as last subsection). The value of $x_{\text {stop }}$ was found to each iteration loop by the statement $x_{\text {stop }}=x_{\text {stop }}+\Delta x$.

The maximum value of $x_{\text {stop }}$ to each value of $\alpha$ determined when the value of $\Delta z$ (the variation of the unknown boundary conditions at $x=0)$ does not change to successful loop with error less than $10^{-8}$. See Fig. 2 for residual error of Eq. (5) obtained by this approach. Table V shows that the initial slope $y^{\prime}(0)$ increases with the increase of $\alpha$ which obtained by this method. Figure 3 shows the obtained $y(x)$ for different values of $\alpha$.

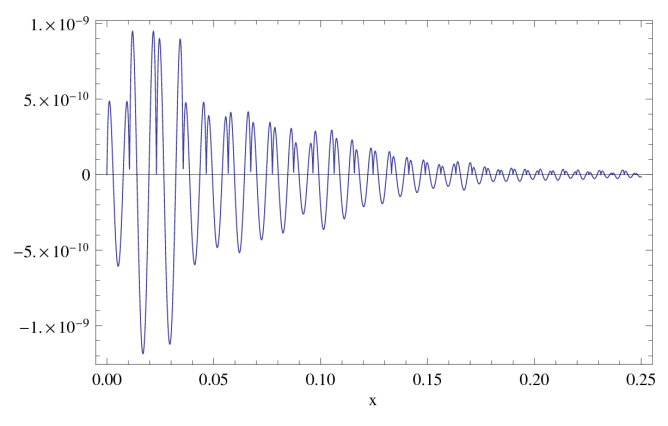

Fig. 2. Residual error of (5) for $\alpha=0.5$.

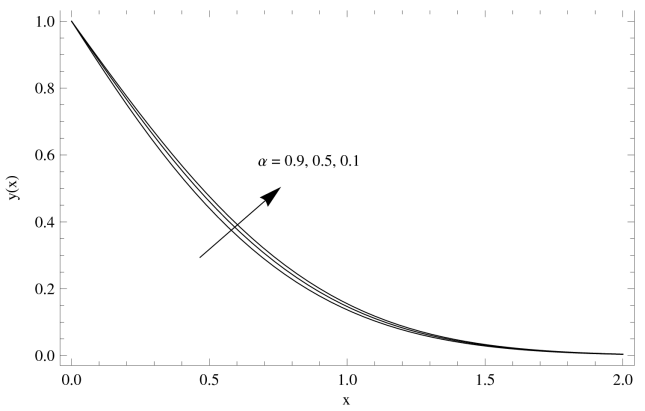

Fig. 3. Approximation of $y(x)$ for different values of $\alpha$.

\section{Concluding remarks}

In this paper, two mentioned powerful and efficient methods are implicit finite-difference Keller-box method and shooting method to solve differential equation of gas flow through a micro-nano porous media. This model is quite valuable in order to its importance in investigating gas-solid processes. By comparison to other previous researcher's numerical solutions, the obtained results in these two methods have provided acceptable approach for nonlinear unsteady gas equation. Consequently we determine the accurate initial slope $y^{\prime}(0) \approx-1.191791$ 
TABLE V

Initial slopes $y^{\prime}(0)$ for various values of $\alpha$.

\begin{tabular}{c|c|c}
\hline \hline$\alpha$ & $x_{\text {stop }}$ & Present method \\
\hline 0.1 & 3.76 & -1.1390073222058392 \\
0.2 & 3.75 & -1.1504756086298318 \\
0.3 & 3.75 & -1.1629415776840015 \\
0.4 & 3.75 & -1.176615782984229 \\
0.5 & 3.74 & -1.1917907719590468 \\
0.6 & 3.74 & -1.2088942932466626 \\
0.7 & 3.73 & -1.2285985979448646 \\
0.8 & 3.73 & -1.2520839101594896 \\
0.9 & 3.72 & -1.2818814468001043
\end{tabular}

for $\alpha=0.5$ with good capturing the essential behavior of $y(x)$.

\section{Acknowledgments}

The authors would like to thanks anonymous referees for their helpful comments.

\section{References}

[1] R.C. Roberts, in: Proc. First US National Congress of Applied Mechanics, Ann Arbor (MI), Eds. E. Sternberg, R.A. Eubanks, M.A. Sadowsky, American Society of Mechanical Engineers, Ann Arbor (MI) 1952 , p. 773

[2] R.E. Kidder, J. Appl. Mech. 24, 329 (1957).
[3] T.Y. Na, Computational Methods in Engineering Boundary Value Problems, Academic Press, New York 1979.

[4] R.P. Agarwal, D. O'Regan, Stud. Appl. Math. 108, 245 (2002).

[5] A.M. Wazwaz, Appl. Math. Comput. 118, 123 (2001).

[6] M. Aslam Noor, S.T. Mohyud-Din, Math. Problems Eng., Article ID 917407 (2008).

[7] K. Parand, A. Taghavi, M. Shahini, Acta Phys. Pol. B 40, 1749 (2009).

[8] A. Taghavi, K. Parand, H. Fani, Int. J. Comput. Math. Sci. 3, 40 (2009).

[9] Y. Khan, N. Faraz, A. Yildirim, World Appl. Sci. J. 9, 27 (2010).

[10] S.T. Mohyud-Din, A. Yildirim, M.M. Hosseini, Int. J. Differ. Equ., Article ID 426213 (2010).

[11] A.R. Rezaei, K. Parand, A. Pirkhedri, J. Comput. Theor. Nanosci. 8, 282 (2011).

[12] H.B. Keller, in: Numerical Solutions of Partial Differential Equations, Vol. II, Ed. B. Hubbard, Academic Press, New York 1971, p. 327.

[13] T. Cebeci, P. Bradshaw, Physical and Computational Aspects of Convective Heat Transfer, Springer, New York 1988.

[14] P.R. Nachtsheim, P. Swigert, "Satisfaction of the asymptotic boundary conditions in numerical solution of the system of non-linear equations of boundary layer type", NASA TND-3004 (1965). 